\title{
Caracterización de las personas con pie diabético. Monterrey, Mexico
}

\author{
Gutiérrez-Valverde Juana Mercedes ${ }^{1, a}$, Gallegos-García Amelia ${ }^{2, b}$, Guevara-Valtier Milton Carlos ${ }^{1, c}$, \\ Vega-Grimaldo Miguel Ángel 1,d, Santos-Flores Jesús Melchor ${ }^{1, \mathrm{e}}$, Paz-Morales María de los Ángeles ${ }^{1, \mathrm{f}}$.
}

\section{RESUMEN}

Objetivos: identificar las características personales, familiares, clínicas y socio económicos de las personas con pie diabético, que estuvieron hospitalizadas en una institución de salud de tercer nivel en Monterrey, Nuevo León durante el año 2009 y 2010. Material y Métodos: fue un estudio descriptivo. La unidad de análisis fueron 275 expedientes clínicos de pacientes con DT2 con diagnóstico de pie diabético. Resultados: el 66,9\% de los expedientes correspondieron al género masculino y un 33,1\% al femenino, con edad media de 59,2 años ( $D E=12,5 ; 31-95)$; el 59,6\% tenían pareja; con una estancia hospitalaria promedio fue 6,4 días $(D E=5,8 ; 1-39)$; el $85,5 \%$ de los participantes cursaron en promedio 5,71 años de educación formal ( $D E=3.8 ; 0-17)$, el $32 \%$ reportaron estar desempleadas, los años de diagnóstico de DT2 en $\mathrm{n}=255$ personas fue de 14.2 años $(D E=9.0 ; 1-40)$; con diagnóstico de pie diabético en $\mathrm{n}=94$ personas $(34,2 \%)$, fue de 3 años $(D E=2.3 ; 1-10)$; destacó la HTA con 22,9\% masculino, 21,4\% femenino con tiempo de diagnóstico de uno a 40 años; el 90\% de los valores de HbAlc fueron superiores a 7\%. En cuanto a los cultivos de úlceras 3,6\% fueron gram+; consumo más el género masculino con $25,8 \%$ de alcohol y $21 \%$ tabaco y solo el $1 \%$ consume marihuana y cocaína; el $43,4 \%$ reportaron DT2 y paterno el $17 \%$; en el tipo de lesión destaca la amputación supracondílea 34\%. Conclusiones: se identificaron factores alarmantes que ubican en riesgo de complicaciones a la persona con DT2, se requiere mayor profundización en el fenómeno estudiado.

PALABRAS CLAVE: Diabetes tipo 2, pie diabético, adultos.

\section{Characterization of patients with diabetic foot. Monterrey, Mexico}

\section{SUMMARY}

Objectives: Identify the personal, relatives, clinics and socio-economic characteristics of the people with diabetic foot who they were hospitalized in a health institution of third level in Monterrey, Nuevo Leon during the 2009 and 2010 years. Material and Methods: It was a descriptive study. In the analysis unit had 275 clinic records of DT2 patients with diabetic foot diagnosis. Results: The $66.9 \%$ of the records corresponded to the masculine gender and the $33.1 \%$ to the female gender, with a media age of 59.2 years $(D E=12.5 ; 31-95)$, the $59.6 \%$ they had a couple, the staying in hospital was 6.4 days $(D E=5.8 ; 1-39)$; the $85.5 \%$ of the participants studied in average 5.71 years of formal education ( $D E=3.8 ; 0-17)$, the $32 \%$ reported being unemployed, the diagnosis years of DT2 in $\mathrm{n}=255$ people were 14.2 years $(D E=9.0 ; 1-40)$; with diabetic foot diagnosis in $\mathrm{n}=94$ people $(34.2 \%)$, it was 3 years $(D E=2.3 ; 1-10)$; the HTA stand out with $22.9 \%$ masculine, $21.4 \%$ female with a diagnosis time of 40 years; the $90 \%$ of the HbAlc values were superior of $7 \%$. With regard to the ulcer culture $3.6 \%$ were gram + ; consumed more the masculine gender with $25.8 \%$ alcohol and $21 \%$ tobacco and just $1 \%$ consumed marihuana and cocaine; the $43.4 \%$ reported DT2 and paternal $17 \%$ in the kind of injury stand out the supracondilea amputation $34 \%$. Conclusions: They were identified alarming factors which place in risk of complication to the DT2 person, it required more deepening in the phenomena studied.

KEYWORDS: Type 2 diabetes, diabetic foot, adults.

Facultad de Enfermería, Universidad Autónoma de Nuevo León. Nuevo León, México.

Hospital Universitario, Universidad Autónoma de Nuevo León. Nuevo León, México.

Doctor en Ciencias de Enfermería, ${ }^{\mathrm{b}}$ Maestra en Ciencias de Enfermería, ${ }^{\mathrm{c}}$ Doctor en Educación, ${ }^{\mathrm{d}}$ Licenciado en Enfermería,

Licenciado en Enfermería, ${ }^{\mathrm{f}}$ Doctor en Educación. 


\section{INTRODUCCIÓN}

La diabetes es un desorden metabólico que afecta diferentes órganos y tejidos caracterizada por hiperglicemia. Esta enfermedad es causada por varios trastornos, incluyendo la baja producción de insulina o por su inadecuado uso (1). La diabetes es una enfermedad crónica degenerativa y se considera un problema de salud pública (2); además de ser la enfermedad de mayor gasto público en salud y de repercusiones en la actividad laboral de las personas quienes la padecen, debido a las complicaciones que se presentan como parte del desarrollo de la enfermedad.

Esta enfermedad se considera la primera causa de demanda hospitalaria en nuestro país ${ }^{(2)}$. Actualmente en México existen 7,3 millones de personas con diabetes lo que representa una prevalencia de $14,42 \%$. En la región Norte donde está localizado Nuevo León la prevalencia es de 12,70\% (3).Así mismo en el Programa de Acción Especifica 2007-2012: Diabetes Mellitus de la Secretaria de Salud (4) afirma que la diabetes es la primera causa de ausentismo laboral, discapacidad, estancias hospitalarias prolongadas, disfunción familiar y mortalidad a consecuencia de las complicaciones micro vasculares y macro vasculares que se desarrollan. Estas complicaciones pueden ser resultado de un inadecuado controlglucémico asociado a estilos de vida no saludables dentro de los que se encuentran la alimentación hipercalórica, poca o nula actividad física y tabaquismo, entre otros.

En las complicaciones micro vasculares, se encuentran la neuropatía periférica, retinopatía y nefropatía. En las macro vasculares están la cardiopatía isquémica, insuficiencia cardiaca, enfermedad vascular cerebral y la enfermedad arterial periférica $(5,6,7)$.Dentro de los dos tipos de complicaciones se encuentra la relacionada con los miembros inferiores, conocida como pie diabético.

El pie diabético comprende una triada etiológica: vascular, neuropática e infecciosa (8), y se caracteriza por la presencia de una o más de las siguientes afecciones: infección, ulceración, destrucción de los tejidos profundos, asociadas con anormalidades neurológicas (pérdida de la sensibilidad al dolor y calor), EAP de diversa gravedad en las extremidades inferiores, trauma (sitios de híper presión), y alteraciones de la biomecánica del pie $(5,7,9)$. De acuerdo a la Federación Internacional de Diabetes (10), alrededor de $15 \%$ de todas las personas con diabetes tipo 2 (DT2) se verán afectadas principalmente por una úlcera a lo largo de su vida u otra complicación del pie. Se calcúla que en todo el mundo la atención por hospitalización es de un $20 \%$ por pie diabético, específicamente lo relacionado con úlceras por debajo de la rodilla. Se estima que cada 30 segundos las persona con diabetes pierden un miembro inferior por causa de la diabetes y se consume hasta el $40 \%$ de los gastos hospitalarios por atender a pacientes con pie diabético.

En Europa la prevalencia de neuropatía es de 49 a $89 \%$ $(11,12)$, de EAP es de 49.3 a $72 \%(11,13)$ y el $61 \%$ de las úlceras se presentan en hombres (14); en África y Asia la neuropatía afecta al $85 \%$ de las personas con diabetes (15) en África la prevalencia de úlceras es de 29,5\% (9) y en Asia es de 32\% (16); en Perú la prevalencia de amputación supracondílea es de 22,2\% (17) y en Venezuela es de 46,9\% (18); en Norteamérica (USA) la prevalencia de neuropatía, ulceración, infecciones y EAP es de 12 a $25 \%$ (8); en poblaciones México-Americanas la prevalencia de neuropatía es de 40,9\% en general (19); el $19 \%$ de EAP se presenta en mujeres (20) y un $75 \%$ de amputaciones en la población general (19).

En México la diabetes es la causa más importante de amputación de miembros inferiores (2). La neuropatía afecta entre 800000 y 1920000 personas por año (21). La prevalencia de neuropatía es de $42,6 \%$ a $55,1 \%$ y de úlcera es de $10,8 \%$ en el Nor oeste del país $(22,23)$. Autores como Castro (24) refieren que la prevalencia de úlceras en personas con DT2 en edades entre 45 y 65 años de edad es de 5,3\% a 10,5\% en la República Mexicana. Además mencionan que una persona con DT2 que ha padecido una amputación en alguno de sus miembros tiene el 50\% de riesgo de perder el otro miembro inferior en los próximos dos a cinco años. De acuerdo a SS, único documento oficial disponible en México con estadísticas relacionadas al pie diabético, refiere que los egresos hospitalarios por esta causa aumentaron en un $10 \%$ y en un $4 \%$ los procedimientos quirúrgicos por amputaciones relacionadas con la diabetes entre el 2004 y 2005 (4).

Ante problemas como el incremento de la DT2 son importantes programas de prevención y/o retardo de complicaciones en las personas que la padecen, mediante intervención de enfermería que ayuden a retrasar el desarrollo de pie diabético y otras complicaciones.

Este estudio contribuirá como base para el desarrollo de intervenciones de enfermería especificas en el manejo del pie, en personas con DT2. Estas intervenciones deben ser a través de técnicas apropiadas para la enseñanza en adultos, considerando el nivel de escolaridad de los pacientes. La literatura muestra la existencia de estudios sobre las características de las personas con pie diabético (EAP, 
neuropatía e infecciosa) en continentes como África y Asia; y en países como Perú,Venezuela, Irlanda, Singapur y España, en donde la prevalencia de pie diabético tiene relación con los años de evolución de la diabetes y con glucemias de $183 \mathrm{mg} / \mathrm{dl}$ y HbA1c $>7 \%(9,12,13,15-18)$. En México se encontraron dos investigaciones que reportaron prevalencias de neuropatía y aspectos generales de úlceras $(22,23)$. Además se encontró un reporte oficial con escasa información sobre el pie diabético SS (4).Por lo que este estudio tuvo el propósito de identificar las características personales, familiares, clínicas y socio económicos de las personas con pie diabético en un hospital de tercer nivel de atención.

\section{MATERIAL Y MÉTODOS}

La unidad de análisis de estudio, fue el expediente clínicode las personas con DT2 que ingresaron a un hospital de tercer nivel del área metropolitana de Monterrey con diagnóstico médico de pie diabético. Se usó el censo de los pacientes que estuvieron hospitalizados en el año 2009 y 2010. El censo reportó 343 expedientes, de los cuales se eliminaron 47 por ser personas con diabetes tipo $1 \mathrm{y} / \mathrm{o}$ que fueron sometidos a algún tipo de amputación por accidente automovilístico; dando un total de 296 expedientes clínicos de los cuales no se tuvo acceso a 21 por razones de logística del departamento de archivo; por lo que la muestra final fue de 275 expedientes clínicos.

Se utilizó una cédula de datos de identificación (CDI) del paciente diseñada para este estudio. Esta cédula consta de: I) Datos de identificación: número de expediente revisado, número de expediente asignado por la institución, fecha de revisión, fecha de ingreso y egreso. II) Datos socio demográficos: edad, género, escolaridad (en años), ocupación, estado civil, código postal. III) Datos clínicos: años de diagnóstico de DT2; años de diagnóstico de pie diabético, nivel de glucosa,HbAlc y creatinina durante la estancia, complicaciones, hábitos de consumo, antecedentes personales y familiares. IV) Lista de afecciones del pie diabético, para ambos miembros inferiores que consta de: necrosis, úlceras, varios tipos de amputaciones y re-amputaciones.

\section{RESULTADOS}

El 66,9\% de los expedientes correspondieron a personas del género masculino y un $33,1 \%$ al femenino. El promedio de edad de los pacientes con pie diabético fue de 59.2 años $(D E=12,5 ; 31-95)$. Se encontró que 164 pacientes $(59,6 \%)$ tenían pareja; en cuanto a la estancia hospitalaria el promedio fue de 6.4 días $(D E=5,8 ; 1-39)$. Con respecto a la educación el $85,5 \%$ de los participantes cursaron en promedio 5.71 años de educación formal $(D E=3,8 ; 0-17)$. Sin embargo 40 de los participantes $(14,5 \%)$ son alfabetos funcionales lo que significa que solo saben leer y escribir sin haber atendido educación formal.

\section{Características generales}

El $32 \%$ (57 personas con pie diabético) en los cuatro niveles educativos reportaron estar desempleadas, la

Tabla 1.Comorbilidades y género de las personas con pie diabético.

\begin{tabular}{|c|c|c|c|c|c|c|c|c|c|c|}
\hline \multirow{4}{*}{ Comorbilidad } & \multicolumn{8}{|c|}{ Género } & \multirow[b]{3}{*}{ Total } & \multirow[b]{4}{*}{$\%$} \\
\hline & \multicolumn{4}{|c|}{ Masculino } & \multicolumn{4}{|c|}{ Femenino } & & \\
\hline & \multicolumn{2}{|r|}{$\mathrm{Si}$} & \multicolumn{2}{|c|}{ No } & \multicolumn{2}{|c|}{$\mathrm{Si}$} & \multicolumn{2}{|c|}{ No } & & \\
\hline & $f$ & $\%$ & $f$ & $\%$ & $f$ & $\%$ & $f$ & $\%$ & $f$ & \\
\hline HTA & 63 & 22,9 & 121 & 44 & 59 & 21,4 & 32 & 11,6 & 275 & 100 \\
\hline Problemas Cardíacos & 11 & 4 & 173 & 62,9 & 6 & 2,1 & 85 & 30,9 & 275 & 100 \\
\hline Problemas Renales & 23 & 8,3 & 161 & 58,5 & 18 & 6,5 & 73 & 26,5 & 275 & 100 \\
\hline Retinopatía & 9 & 3,2 & 175 & 63,6 & 2 &, 7 & 89 & 32,3 & 275 & 100 \\
\hline Ceguera & 3 & 1 & 181 & 65,8 & 2 &, 7 & 89 & 32,3 & 275 & 100 \\
\hline Cáncer & 1 & , 3 & 183 & 66,5 & 0 & 0 & 91 & 33 & 275 & 100 \\
\hline Obesidad & 7 & 2,5 & 177 & 64,3 & 5 & 1,8 & 86 & 31,2 & 275 & 100 \\
\hline Artritis & 1 & ,3 & 183 & 66,5 & 1 &, 3 & 90 & 32,7 & 275 & 100 \\
\hline Enfisema & 1 &, 5 & 183 & 99,4 & 0 & 0 & 0 & 0 & 184 & 100 \\
\hline Alzhéimer & 0 & 0 & 0 & 0 & 1 & 1 & 90 & 98,9 & 91 & 100 \\
\hline Parkinson & 0 & 0 & 0 & 0 & 1 & 1 & 90 & 98,9 & 91 & 100 \\
\hline
\end{tabular}

Fuente: Expedientes Clínicos. 
Tabla 2. Características bioquímica de las personas con pie diabético

\begin{tabular}{lrrrrr}
\hline \multicolumn{1}{c}{ Bioquímicos } & $\mathrm{n}$ & $M$ & $D E$ & Mínimo & Máximo \\
\hline HbA1c (\%) & 240 & 9,2 & 2,5 & 5 & 18 \\
Glucosa (mg/dl) & 269 & 232,2 & 108,4 & 66 & 671 \\
Creatinina (mg/dl) & 270 & 1,5 & 1,8 & 0,4 & 14 \\
\hline
\end{tabular}

Fuente: Expedientes Clínicos.

mayoría de estas solo tiene nivel de primaria 27 (15,2\%). En relación a la ocupación de los participantes, el género masculino refirió haber trabajado a lo largo de su vida y el 1,4\% reportaron haberse dedicado al hogar. En cuanto al género femenino registraron tener menor actividad laboral a lo largo de su vida, donde destaca que solo el $1 \%$ de las mujeres están pensionadas, un 7\% desempleadas y $31,2 \%$ se dedica al hogar. El 50,6\% corresponden a un nivel socioeconómico medio bajo y el $6,9 \%$ pertenecen al nivel marginal.

\section{Antecedentes clínicos.}

El tiempo de evolución de la DT2 y el diagnóstico de pie diabético fueron reportados en años o en meses. En $n=$ $255(92,7 \%)$ el promedio de años de diagnóstico de DT2 fue de 14,2 años $(D E=9.0 ; 1-40)$, y el resto de la población $\mathrm{n}=20(7,3 \%)$ reporto una media de 2,4 meses con el diagnóstico de DT2 $(D E=2,4 ; 1-10)$. El promedio de años de diagnóstico de pie diabético en $\mathrm{n}=94$ personas $(34,2 \%)$, fue de 3 años $(D E=2,3 ; 1-10)$, y en $\mathrm{n}=181$ personas $(65,8 \%)$ los meses de diagnóstico de pie diabético fue 2,7 meses $(D E=3,0 ; 1-18)$.

En la tabla 1, se muestran las comorbilidades de las personas con pie diabético donde destaca la HTA con mayor frecuencia, seguida por los problemas renales en ambos sexos. Con respecto al tiempo de diagnóstico médico de las comorbilidades se reportaron en años y meses. La HTA en las personas con pie diabético reportaron un máximo de 40 años con la comorbilidad, seguida por las cardiopatías con un máximo de 10 años.En la tabla 2, se presentan las características bioquímicas de las personas con pie diabético. El 90\% de los valores de HbA1c reportados en el expediente clínico fueron valores superiores a 7\%.De los 17 pacientes que presentaron úlceras solo 13 autorizaron que se les tomara cultivo de la lesión. El 3,6\% fueron gram $+, 0,7 \%$ gram- y $0,4 \%$ no pudo ser identificada la bacteria.

Con respecto al consumo de alcohol, tabaco, marihuana y cocaína de las personas con pie diabético de las que destaca el género masculino que consume alcohol 25,8\% y tabaco $21 \%$ y solo el $1 \%$ consume marihuana y cocaína.
El $43,4 \%$ de los participantes reportaron tener antecedentes familiares de DT2, seguido por el $17 \%$ con antecedentes paternos.

\section{Tipos de lesiones.}

El $27 \%$ presentó dos afecciones (neuropatía y EAP) y los que presentaron tres afecciones (combinación de neuropatía, EAP y algún tipo de amputación). Los tipos de lesiones que se observaron con mayor frecuencia fueron: la amputación supracondílea $n=93(34 \%)$, seguido de amputación de dedos $n=53(19 \%)$, las cuales se presentaron más en el primer, segundo, tercero y cuarto ortejo, la amputación transtibial $n=42(15,2 \%)$, celulitis $n=22$ (8\%) se presentó con más frecuencia en los dedos seguido del talón, de igual proporción la plantar, dorsal y por último la pretibial. Las úlceras $n=17(6,2 \%)$, se presentaron más en los dedos, seguido de la planta, después del talón y por último en el dorso.

\section{DISCUSIÓN}

Los resultados del presente estudio permitieron identificar las características personales, familiares, clínicas y socio económicos de las personas con DT2 que presentaron pie diabético en alguna de sus manifestaciones. La presencia de pie diabético es variable dependiendo de la región geográfica y el nivel socioeconómico. En esta muestra, el pie diabético fue más frecuente en pacientes de género masculino 184 (66,9\%) que en el femenino 91 (32,9\%), esto coincide con lo reportado por Camacho (22); Colom (11);Martínez (14); Nather (16); Romero-Guarecuco (18) y Vidal (17). En cuanto a la edad de presentación de la enfermedad comprende un rango de edad de 31 hasta 95 , esto concuerda con la literatura encontrada con rango de 24 hasta 91 años (16).

Es importante destacar que un $17,1 \%$ de los pacientes investigados son viudos; lo cual crea un factor que interfiere en el manejo de la DT2, pues en algunos casos la pérdida de su pareja provoca alteraciones de salud, tales como depresión, desánimo y pérdida del deseo de vivir (25).

En cuanto a las estancia hospitalaria reportada es similar 
a la literatura ya que el promedio fue de 6,4 días, con estancia menor de un día de hospitalización, encontrándose que la frecuencia mayor fue 39 días. La literatura habla de un periodo de estancia de entre 7 a 35 días (14).

En relación a la escolaridad, la mayoría posee enseñanza primaria o incompleta $160(58,18 \%)$, lo cual está de acuerdo con literatura encontrada con 59,3\%(25), estas cifras difieren con la investigación de Adigun y Olarinoye (9) donde la primaria tiene un $19 \%$. Ya que la baja situación socio económica está relacionada con la baja escolaridad (26). Con respecto a la ocupación en la mayoría se dedican al hogar 90 (32,7\%). Estos datos son congruentes con las personas de género femenino con nivel bajo de escolaridad.

En relación a los antecedente personales de DT2, el promedio de años de diagnóstico en este estudio fue de 14,2 años, esto se relaciona que a mayor tiempo de diagnóstico, peor es el resultado, hasta que tenga que realizarse una amputación (13). El resto reporto un promedio de 2.4 meses con el diagnóstico de DT2, quedando demostrado que cada vez es más común detectar complicaciones al momento del diagnóstico $(22,25)$, por el diagnóstico tardío se detectan otras complicaciones (25).

El promedio de años de diagnóstico de pie diabético fue de 3 años, y en meses de diagnóstico de pie diabético fue de 2,7 meses. Esto refleja que un gran número de pacientes acude al servicio hospitalario a solicitar ayuda hasta que la lesión está muy avanzada (13), ya que en nuestro estudio hubo una alta prevalencia de amputación mayor $49,6 \%$ y menor en un $24,7 \%$ y es similar con la literatura (18) la amputación mayor se presenta en un $46,9 \%$ pero difiere la amputación menor con 1,2-42\% $(11,18,13)$. En los años de escolaridad y género de las personas con pie diabético se observa que el mayor porcentaje de la población concluyó sus estudios de primaria, fueron 102 (37\%) de los cuales $70(38 \%)$ son hombres y $32(35,1 \%)$ son mujeres, son datos relevantes para implementar métodos de enseñanza que faciliten la comprensión sobre el cuidado de los pies (26).

En la comorbilidad del pie diabético, donde destaca la HTA con un $34,2 \%$ en hombres y en mujeres con un $64,8 \%$, dado que la HTA es un factor de riesgo para desarrollar complicaciones micro vasculares y macro vasculares, estos resultados son superiores a lo reportado donde señala de un 30 a un 50\% (27).La HTA es una de las comorbilidades más comunes de la diabetes se presenta hasta el 50\% (5). La hipertensión en años de diagnóstico se presenta más con una media de 10,9 años $(D E=9$; 1-40). Dado que la presencia de complicaciones como retinopatía, nefropatía, enfermedades macro vasculares aumentan la probabilidad de presentar lesiones en los pies (5). En cuanto a las complicaciones renales, en este estudio se presenta $32,2 \%$ estos datos son superiores a lo reportado $(5,23)$, el problema renal se presenta en un 10 al $25 \%$ de los pacientes con DT2.

Al analizar los valores de HbA1c, se identificó a 70 (29\%) de los pacientes presentaban valores superiores de $7 \%$, siendo la media de $8,6 \%(D E=2.5 ; 5-18)$. Considerando que la $\mathrm{HbA} 1 \mathrm{c}$ es capaz de pronosticar el riesgo de desarrollar varias complicaciones crónicas de la diabetes ya que se recomienda verificar en todos los pacientes para obtener el control metabólico de la enfermedad y el ajuste de dosis necesario de acuerdo a los resultados ${ }^{(16,22)}$.En las glucemias, se mostró a 235 personas con pie diabético $(87,3 \%)$ con glucemias arriba los parámetros normales de $70 \mathrm{a} 118 \mathrm{mg} / \mathrm{dl}$. Se obtuvo la media de glucosa es $232,2 \mathrm{mg} /$ $\mathrm{dl}(D E=108,4 ; 66-671)$, son cifras constantes que provocan más complicaciones de la DT2 (9). En la creatinina, se identificó a 69 pacientes con pie diabético $(25,5 \%)$ con creatinina arriba de los valores normales $(0,8$ a $1,4 \mathrm{mg} /$ dl), con una media de $1.53 \mathrm{mg} / \mathrm{dl}(D E=1,8 ; 4-14)$, son cifras que marcan daño en los riñones (28).

De los cultivo que se tomaron para administrarles el antibiótico adecuado, destacaron $10(3,6 \%)$ de gram+, fue una cifra inferior al estudio de (14) con cifras de $55 \%$ en la mayoría de las úlceras. En cuanto a la ubicación de las úlceras se presentaron con más frecuencia en dedos, seguido de las plantas, después en talón y por último en dorso concordando con la investigación (29).En el consumo de varias sustancias, de las que destaca $25,8 \%$ de consume alcohol y $21 \%$ consumo de tabaco son cifras altas comparadas con estudidos realizados en Singapur, donde consumen alcohol un $9,2 \%$, esto significa que es un factor predisponente para la aparición de problemas de los pies y el tabaco se presentó en un $15,9 \%$ donde el fumar es un factor de riesgo de complicaciones (16).

Al investigar los antecedentes familiares, se encontró que el $43,4 \%$ de las personas con pie diabético tenían al menos un familiar con diabetes, la herencia como un factor de riesgo, este resultado es superior a lo reportado por los autores que señalan un $38 \%$ (30). La muestra tuvo una sola afección de neuropatía con $2 \%$, EAP $3.2 \%$, tres afecciones $1 \%$, combinada con EAP y neuropatía $1 \%$ se observan cifras inferiores a lo reportado por Touceda(13) con una sola afección mostro una prevalencia de neuropatía 13,1\%, 
EAP 49,3\%, combinada EAP y neuropatía 9,1\% (13).

\section{REFERENCIAS BIBLIOGRÁFICAS}

1. American Diabetes Association. Diagnosis and classification of Diabetes. Diabetes Care. 2010; 33(1),562-569

2. Secretaria de Salud. Programa Nacional de Salud: Por un México sano: construyendo alianzas para una mejor salud. 2007-2012. México D F: Secretaria de Salud; 2007 (Citado el 21 noviembre 2015). Disponible en: http://portal.salud.gob.mx/descargas/pdf/pns_version_ completa.pdf

3. Villalpando S, Rojas R, Shamah-Levy T, et al. Prevalence and distribution of type 2 diabetes mellitus in Mexican adult population. A probabilistic survey. Revista de Salud Pública México, 2010; 52(1):19-26.

4. Secretaria de salud. Programa de acciones específicas de Diabetes Mellitus. 2007-2012. México DF: Secretaria de Salud; 2008 (Citado el 21 noviembre 2015) Disponible en: http://www.cenave.gob.mx/ progaccion/diabetes.pdf

5. Asociación Latinoamericana de Diabetes. Diagnóstico, control y tratamiento de la Diabetes Mellitus Tipo 2. ALAD 2006 (Citado el 21 de noviembre 2015) Disponible en: http://revistaalad.com.ar/guias/ GuiasALAD_DMTipo2_v3.pdf_

6. Deshpande AD, Harris MH, Schootman M. Epidemiology of diabetes-related complications. Physical Therapy. 2008; 88(11):1254-1264.

7. Sánchez ML, Cruz IG, Pérez MG, Plata AI, Sánchez AR. Complicaciones macro vasculares del paciente diabético. Pie diabético. Medicine. 2008; 10(17):11101124.

8. Andersen ChA, Roukis TS. El pie diabético. Surgical Clinics of North America. 2007;87(1):1149-1177.

9. Adigun I, Olarinoye J. Foot complications in people with diabetes: Experience with 105 Nigerian Africans. The Diabetic Foot Journal. 2008; 11(1):36-42.

10.Federación Internacional de Diabetes. Perspectivas mundiales de la diabetes. Diabetes Voice. 2005; 50(1):1-48.

11.Colom C, Aulinas A, Pérez A, et al. Cambios en el perfil del paciente diabético hospitalizado en una sala de cirugía vascular y de los procedimientos quirúrgicos utilizados en 1985 y 2007. Avances en Diabetología. 2008; 24(6):481-487.

12.Kelly S, Dolan C, Hurley L, et al. Prevalence of diabetic foot complications in the west of Ireland: A pilot study. The Diabetic Foot Journal. 2010;13(2):8289.
13.Touceda SR, Pérez SG, Barreiro DL, García MP, Vázquez RM, López DL. Incidence of Diabetic Foot in the Podiatry Clinic of the University of La Coruña. Internacional de Ciencias Podológicas. 2010;4(2):9-19.

14. Martínez DG, Ramírez CA, Campillo AS, Aguayo JA. Infecciones del pie diabético. Prevalencia de los distintos microorganismos y los antimicrobianos. Enfermedades infecciosas microbiología clínica. 2009;27(6):317-332.

15. Abbas ZG, Lutale JK, Archibald LK. Diabetic foot ulcers and Ethnicity in Tanzania: a contrast between African and Asian populations. International Wound Journal. 2009; 6(2):124-131.

16. Nather B, Yan L, Li X, Bee S, Huak Y, Hung P. SocioEconomic factors of diabetics with and without foot problems in Singapore. Diabetic Foot Problems. 2010; 2(1):15-28.

17.Vidal GD. Factores de riesgo de amputaciones en el pie diabético. Rev Soc Peru Med Interna. 2010; 23(4):145149.

18. Romero-Guarecuco J, Hernández J, Tovar A, Monitel M, Sánchez J. Morbilidad de pacientes con pie diabético ingresado al servicio de cirugía del Hospital Universitario "Dr. Alfredo Van Grieken" coro, estado Falcón. Venezuela de Cirugia. 2004;60(1): 21-26.

19.Lavery L, Armstrong D, Wunderlich R, Tredwell J, Boulton A. Diabetic foot Syndrome, Evaluating the prevalence and incidence of foot pathology in Mexican-Americans and non-Hispanics white form a diabetes disease management cohort. Diabetes Care. 2003; 26(5):1435-1438.

20.Ostchega Y, Paulose-Ram R, Dillon Ch, Gu Q, Hughes J. Prevalence of peripheral arterial disease and risk factors in persons aged 60 and older: Data from the National Health and Nutrition Examination Survey 1999-2004. J Am Geriatr Soc. 2007;55(4):583-9.

21.Flores S, León M, Torres M, Reyes F, Serpa J, Ríos A. Manejo farmacológico del dolor neuropático. Colombiana de Anestesiología. 2009; 37(4):356-672.

22. Camacho JL. Prevalencia de neuropatía en pacientes con diabetes mellitus tipo 2, atendidos en la Clínica Hospital del ISSSTE en Mazatlán, Sinaloa. Especialidades Médico Quirúrgicas. 2011;16(2):71-74.

23.Sabag ER, Álvarez AF, Celiz SZ, Gómez AA. Complicaciones crónicas en la diabetes mellitus. Prevalencia en una unidad de medicina familiar. Medicine. 2006; 44(5):415-421.

24.Castro G, Liceaga G, Arnoja A, et al. Guía clínica basada en evidencia para el manejo del pie diabético. Medicina Interna de México. 2009; 25(6): 483-526. 25.Miyar L, Zanetti M, Teixeira C. Características 
sociodemográficas y clínicas de una población Diabética en el nivel primario de atención a salud. Enfermagem. 2007; 15:1-7.

26. Medina SH, García CR, Gómez VM, Celaya FG. Cuidados de los pies en usuarios que viven con diabetes en el estado de México: bases para la sistematización de la asistencia de enfermería. Enfermagem. 2011; 2(1): 23-27.

27. Ministerio de Salud. Guía Clínica Diabetes Mellitus tipo 2. Santiago de Chile: Ministerio de Salud; 2010.

28. Bazarin H. Approach to the patient with renal. Philadelphia: Cecil. Medicine-Saunders Elsevier; 2007.

29. Boulton JA, Cavanagh RP, Rayman G. Pie diabético. $1^{\mathrm{a}}$ ed. Colombia: El Manual Moderno; 2007.

30. Ministerio de Salud. Guía Clínica Diabetes Mellitus Tipo 2; Chile, Santiago: Ministerio de Salud, 2010. 31. Stumvoll D, Haeften V. Diabetes tipo 2. Lancet. 2005;365(9467):1333-46.

\section{Correspondencia:}

Juana Mercedes Gutiérrez Valverde,

Correo electrónico: juana.gutierrezv@uanl.mx

Fecha de Recepción: 27 de noviembre del 2015

Fecha de aceptación: 03 de diciembre del 2015 